\title{
Mended But Not Healed - Somatic and Mental Health 10 Years after Leg Lengthening with Ilizarov
}

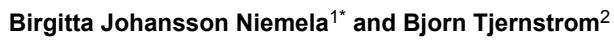 \\ ${ }^{1}$ Department of Neuroscience, Child and Adolescent Psychiatry, Uppsala, Sweden \\ ${ }^{2}$ Department of Surgical Sciences, Department of Pediatric OrthopedicsUppsala University Hospital, Uppsala, Sweden
}

"Corresponding author: B Johansson Niemela, Department of Child and Adolescent Psychiatry, Uppsala University Hospital, SE-75185 Uppsala, Sweden, Tel: +46 18-6114284; Fax: +46 18-6115853; E-mail: birgitta.johansson.niemela@akademiska.se

Rec date: Jan 24, 2014, Acc date: Feb 18, 2014, Pub date: Feb 25, 2014

Copyright: @ 2014 Niemela BJ, et al. This is an open-access article distributed under the terms of the Creative Commons Attribution License, which permits unrestricted use, distribution, and reproduction in any medium, provided the original author and source are credited.

\begin{abstract}
Objective: To study the somatic and mental health in patients who had leg lengthening (LL) in a 10-year follow-up and to compare it with the 1 year-follow-up. Earlier studies have demonstrated severe maladaptive reactions in children and adolescents on a short term basis but also that they tolerate it without sustained psychological impact. Cohort sequential longitudinal studies, in which individuals from different age groups are followed over time, are needed to tease apart aging and cohort effects. Methods: This was a prospective study with 28 patients, mean age of 20, who had undergone leg lengthening (LL) between 1997-2005 at the Department of Pediatric Orthopedics, Uppsala University Hospital. The somatic health was studied by a structured medical assessment of the reconstructed leg and an interview focused on the patient's experiences of surgery, outcome and function. The mental health was studied by Beck depression- and the State-Trait Anxiety Inventories and a specially designed questionnaire for patient with LLI. Results: When the patients rated their mental health, 20 were within a normal level and 7 had symptoms of depression. Patients' self-esteem was on a median level. Those who had scored highly on the depression inventory also had high levels on both State- and Trait anxiety scale. Conclusion: One fourth of the patients reported themselves to be depressed at the 10-year follow-up, while $7 \%$ of the patients were depressed one year after LL. Self-esteem results for the cohort were on a median level, both one and ten years after LL. Interview data demonstrated that the majority of patients were preoccupied with their LLI and more psychological support during the lengthening period would have been desirable. From available data we cannot conclude that the leg lengthening per se caused the depressive symptoms in the patients.
\end{abstract}

Keywords: Leg lengthening; Adolescents; Mental health; Somatic health

\section{Introduction}

The Ilizarov Technique has become an established method in the treatment of children with significant shortening and angular deformities of the extremities [1-5]. Earlier studies have demonstrated severe maladaptive reactions in children and adolescents undergoing leg lengthening (LL) [6,7]. However it is suggested that, allthough the Ilizarov treatment is demanding for adolescents, it is one that they tolerate without sustained psychological impact [8-10]. Moraal et al. concluded that the patients still have physical limitations but that they appear to have normal psychosocial functioning and self-esteem. Residual leg length inequality (LLI) of more than $2 \mathrm{~cm}$ remains important even after long-term follow-up, as these patients report lower quality of life [11]. Several of the patients have complex deformities of limb and foot and/or neurological deficits that could not always be corrected by the LL. This could of course effect the patients opinion of the procedure and his/hers well-being.

Cohort sequential longitudinal studies, in which individuals from different age groups are followed over time, are needed to tease apart aging and cohort effects. Robins and Trzesniewski [12] asked the question: "will all older individuals develop lower self-esteem or just the particular cohort of individuals who experienced the Great Depression and other life events unique to that cohort?" (p.161). Undergoing leg lengthening (LL) could be one such life event for the cohort in the present study, which could affect self-esteem. The surgical intervention could help the patient by reducing impairment in daily life and thereby improving mental health. On the other hand, the impact of surgery could have an immediate negative effect on mental health defined more like State-Anxiety (relatively transitory). The sensitivity of the State-Anxiety to environmental stress has been repeatedly demonstrated in research on emotional reaction to surgery $[13,14]$. Typically, State-Anxiety scores rise immediately prior to surgery and decline as patients recuperate. In contrast, Trait-Anxiety scores are essentially the same before and after surgery and do not appear to be influenced by the stress of surgical procedures. Although Trait-Anxiety scores do not predict differences in emotional reactions to physical danger, persons high in Trait-Anxiety generally respond with greater elevations in State-Anxiety to threats to self-esteem than do low Trait-Anxiety individuals [12,15].

The present study, where LL patients functioned as their own controls, focused on the mental health and somatic function on a longterm basis. The patients had previously taken part in a prospective study with a preoperative assessment and a one-year follow-up [10]. The result of that study was that the patients before surgery expressed more depressive and anxiety symptoms than the control group of normal school children. One year after surgery the patients had significantly improved their mental health concerning anxiety and depression symptoms. The present design gave the opportunity to study the impact of surgery on both the patient and the parent group in a 10-year follow-up. We included the parents since their level of stress were high before their child's surgery. In order to better 
understand the dynamic factors affecting mental health, we tested for a correlation between patients' and parents' anxiety levels.

We hypothesized that mental health would have been further improved by increased function and improved appearance due to the LL procedure. A 1-year follow-up is short and regarding LL, which is a very prolonged procedure, it would probably mainly reflect the reaction to the procedure. A follow-up of at least 2 years is required to evaluate if the surgical procedure has succeeded. As the rehabilitation after LL is drawn-out we aimed at a long follow-up time. We also tested agreement between the instruments for depression and anxiety. In clinical settings with focus on somatic issues surgeons need more information on how their patients rate their emotional stress.

\section{Methods}

\section{Participants}

We studied the somatic and mental health of 28 patients, $14 \mathrm{men} / 14$ women with a mean age of 20.4 years $(\mathrm{sd}=4.0,14-28)$, who in average $10.6(\mathrm{sd}=2.7$, range $=7-14)$ years earlier, had undergone an Ilizarov LL of 35 segments (20 tibias and 15 femurs) between 1997-2005 at the Department of Pediatric Orthopedics, Uppsala University Hospital.

One single surgeon performed the reconstructive surgery and one single psychologist performed the psychological measurement. There were 30 patients in the study, but one patient died of unrelated causes and one did not partake in the follow up. Preoperative medical condition for the participating patients is shown in Table 1.

\begin{tabular}{|l|l|}
\hline Congenital & 17 \\
\hline Post infection & 3 \\
\hline Neurofibromatosis (overgrowth) & 2 \\
\hline Neurologic & 3 \\
\hline Perthes & 1 \\
\hline PEVA & 1 \\
\hline Short stature & 1 \\
\hline
\end{tabular}

Table 1: Diagnosis $n=28$

\section{Measures}

The medical examination was a structured medical assessment of the reconstructed leg and an interview focused on the patient's experiences of surgery, outcome and function.

Beck Depressive Inventories (BDI-II) were used for self- assessment of mental health $[16,17]$. BDI-II contains 21 items, with a total score ranging from $0-63$, where each item is scored $0-3$. Total score of $0-13$ indicate minimal depression, 14-19 mild depression, 20-28 moderate depression and 29-63 severe depression. The Inventories by Beck has been standardized on a large sample of Swedish adolescents, which gives a good base for comparison of data concerning rate of depression in adolescents with or without psychiatric problems. The State-Trait Anxiety Inventory (STAI) was used to measure parental and patient anxiety [15]. The STAI contains 20 items, with a total score ranging from 0-60, mean normal values are reported to range from 35-39 (SD 10-12) with the somewhat higher levels for adolescents compared to adults. The sensitivity of the STAI anxiety scale to environmental stress has been demonstrated repeatedly in research on emotional reactions to surgery.

These self-rating scales are well- defined instruments with defined categories to measure depression and anxiety. The mental health measures at the 1-year follow-up are described in the original article by Niemelä et al. [10].

Condition specific topics were studied by Leg Length Inequality Youth Questionnaire, (constructed by Niemelä [18]. The questionnaire was used in an individual interview situation. The following questions were selected to present especially important themes for this cohort of patients: When did you understand that you looked different, were different from other children? What emotional reactions did you feel after surgery? Pain experiences associated with the LL procedure? Did you get a lot of attention because of your leg length inequality (LLI)? How often do you think of your LLI? If you rate your self-esteem on a scale 1-9 at the ages of 6 years, 8-10 years and 13-19 years of age on a scale 1-9 what would it be? What made your self-esteem/your view of yourself waver? Do you perceive yourself as having been socially isolated (staying at home more often, mostly seeing your classmates and not so many others at your age) on account of your LLI?

\section{Procedure}

The medical and psychological assessments took place on the same day at Uppsala University Hospital. The somatic health was first studied by the surgeon (BT) at the Department of Pediatric Orthopedics. The psychological data were collected by the psychologist (BJN) from interviews and self-rating scales. The assessment with adolescents and parents took place at the Child and Adolescent Psychiatric clinic. Parents and adolescents filled in selfrating scales separately at the same occasion.

\section{Statistical analysis}

1-year and 10-year follow-up effects for State and Trait were compared with Repeated measure ANOVA where baseline (before surgery) was set as reference category. As depression symptoms were measured with CDI at baseline/1-year follow-up and BDI-II at 10-year follow-up Wilcoxon test were used for measuring changes in depression effect. T-test was performed for comparing age differences. Pearson's correlation coefficient was used to investigate agreement between variables. A significance level of $5 \%$ was used. All analysis were executed with SPSS version 21.

\section{Ethics}

The study (2009/356) was approved by the regional ethics committee. The participants received a letter with information about the study; and they then entered their information regarding when they could come to an appointment, and finally signed a letter with full consent to enter the study.

\section{Results}

\section{Somatic Health}

10 patients had more than 1 lengthening, and 25 one or more surgical procedures before or after LL. Average lengthening was 6,5 $(2-13) \mathrm{cm}$. 
Citation: Niemela BJ, Tjernstrom B (2014) Mended But Not Healed - Somatic and Mental Health 10 Years after Leg Lengthening with Ilizarov. J Depress Anxiety 3: 151. doi:10.4172/2167-1044.1000151

Page 3 of 7

\section{Socio-economic situation}

All of the patients $n=28$ had meaningful social activities such as schooling (17) or work (11). 10 lived with their parents and 18 had

\begin{tabular}{|l|l|}
\hline Satisfied or very satisfied with the length & 26 \\
\hline Satisfied or very satisfied with the cosmesis & 16 \\
\hline Ordinary shoes & 24 \\
\hline Walking distance exceeding $3 \mathrm{~km}$ & 20 \\
\hline Decreased ankle motion and 2 decreased knee motion & 12 \\
\hline$>1,5 \mathrm{~cm}$ LLI & 3 \\
\hline$>1,5 \mathrm{~cm}$ LLI waiting for another lengthening & 2 \\
\hline Amputated on the long side due to risk for malign transformation of neurofibromatosis & 2 \\
\hline
\end{tabular}

Table 2: Somatic health $(\mathrm{n}=28)$

\begin{tabular}{|c|c|c|c|c|c|c|c|c|c|c|c|c|c|c|c|c|c|c|c|c|}
\hline A & B & C & D & $E$ & $\mathrm{~F}$ & G & $\mathrm{H}$ & 1 & $J$ & $\mathrm{~K}$ & L & M & $N$ & O & $P$ & Q & $\mathrm{R}$ & $S$ & $\mathrm{~T}$ & U \\
\hline 1 & M & 8 & 12 & Cong & $x$ & $x$ & 13 & 12 & 3 & 1 & 45 & S & 4 & 4 & 1 & $x$ & 0 & 1 & $A+K$ & 1,5 \\
\hline 2 & $\mathrm{~F}$ & 9 & 11 & Cong & $x$ & $x$ & 11 & 8 & 3 & 1 & 43 & S & 4 & 4 & 4 & $x$ & $x$ & 2 & A & $4 \#$ \\
\hline 3 & $\mathrm{~F}$ & 7 & 6 & Cong & & $x$ & 6 & 14 & $x$ & $x$ & 36 & S & 4 & 3 & 0 & $x$ & $x$ & 0 & 0 & 1,5 \\
\hline 4 & $\mathrm{~F}$ & 9 & 10 & Cong & $x$ & $x$ & 10 & 13 & $\mathrm{x}$ & 9 & 19 & M & 4 & 2 & 0 & $x$ & 0 & 1 & A & 0 \\
\hline 5 & $\mathrm{~F}$ & 7 & 5,5 & Cong & & $x$ & 5,5 & 14 & $x$ & 11 & 16 & M & 5 & 5 & 0 & $x$ & $x$ & 0 & 0 & 0 \\
\hline 6 & $\mathrm{~F}$ & 14 & 0 & Short & $x 2$ & & 10 & 7 & 8 & 2 & 15 & M & 5 & 3 & 0 & $x$ & 0 & 0 & A & 0 \\
\hline 7 & $M$ & 15 & 5 & Perthes & & $x$ & 4 & 9 & 27 & 19 & 14 & M & 4 & 4 & 1 & ortosis & 0 & 1 & A & HP \\
\hline 8 & $\mathrm{~F}$ & 16 & 4 & Neurolog & $x$ & & 4 & 9 & 4 & 3 & 11 & $\mathrm{~N}$ & 3 & 4 & 1.5 & ortosis & 0 & 2 & 0 & 1,5 \\
\hline 9 & $M$ & 14 & 6 & Cong & & $x$ & 6 & 13 & $x$ & 0 & 10 & $\mathrm{~N}$ & 5 & 3 & 0 & $x$ & 0 & 0 & A & 0 \\
\hline 10 & $\mathrm{~F}$ & 15 & 4 & St post inf & & $x$ & 4 & 11 & 9 & 6 & 8 & $\mathrm{~N}$ & 4 & 2 & 0 & $x$ & $x$ & 0 & A & 0 \\
\hline 11 & $\mathrm{~F}$ & 13 & 6 & Cong & $\mathrm{x}$ & & 6 & 10 & 4 & 4 & 8 & $\mathrm{~N}$ & 5 & 2 & 0 & $\mathrm{x}$ & 0 & 0 & 0 & 0 \\
\hline 12 & $\mathrm{~F}$ & 14 & 5 & Cong & $x$ & & 5 & 14 & $x$ & 5 & 7 & $\mathrm{~N}$ & 5 & 4 & 0 & $\mathrm{x}$ & $x$ & 0 & 0 & 0 \\
\hline 13 & M & 8 & 6 & Neurofibr & $x$ & & 7 & 14 & $x$ & 9 & 7 & $\mathrm{~N}$ & 5 & 2 & 0 & $x$ & $x$ & 0 & 0 & KA \\
\hline 14 & $M$ & 12 & 5 & PEVA & $x$ & & 5 & 8 & 10 & 17 & 6 & $\mathrm{~N}$ & 4 & 5 & 0 & $x$ & 0 & 0 & A & $2 \mathrm{PE}$ \\
\hline 15 & $\mathrm{~F}$ & 9 & 6 & Cong & & $x$ & 5 & 12 & $x$ & 1 & 5 & $\mathrm{~N}$ & 5 & 2 & 0 & $x$ & 0 & 2 & A & 0 \\
\hline 16 & M & 14 & 10 & St post inf & $x$ & & 8 & 9 & 4 & 3 & 5 & $\mathrm{~N}$ & 4 & 5 & 1 & $x$ & $x$ & 0 & A & $\mathrm{DH}$ \\
\hline 17 & $M$ & 8 & 6 & Neurofibr & $x$ & & 6 & 14 & $x$ & 8 & 5 & $\mathrm{~N}$ & 5 & 3 & 0 & $x$ & 0 & 0 & 0 & $\mathrm{KA}$ \\
\hline 18 & M & 11 & 5 & Neurolog & $x$ & & 4 & 9 & 10 & 4 & 4 & $\mathrm{~N}$ & 4 & 5 & 0 & ortosis & 0 & 1 & 0 & 1 \\
\hline 19 & $M$ & 11 & 6 & Cong & & $x$ & 6 & 14 & $x$ & 1 & 3 & $\mathrm{~N}$ & 4 & 3 & 0 & $x$ & $x$ & 0 & 0 & 1 \\
\hline 20 & $M$ & 16 & 4 & Cong & $x$ & & 4 & 14 & $x$ & 1 & 1 & $\mathrm{~N}$ & 5 & 4 & 0 & $x$ & 0 & 0 & 0 & 0 \\
\hline 21 & $M$ & 16 & 5 & Neurolog & $x$ & & 5 & 13 & 3 & 1 & 1 & $\mathrm{~N}$ & 4 & 5 & 0 & $x$ & $x$ & 2 & 0 & 0 \\
\hline 22 & $\mathrm{~F}$ & 13 & 6 & Cong & $x$ & $x$ & 6 & 9 & 4 & 5 & 0 & $\mathrm{~N}$ & 5 & 4 & 0 & diff sice & 0 & 0 & A & 0 \\
\hline 23 & $\mathrm{~F}$ & 15 & 3 & Cong & & $x$ & 3 & 9 & 4 & 2 & 0 & $\mathrm{~N}$ & 5 & 1 & 0 & $\mathrm{x}$ & $x$ & 0 & 0 & 0 \\
\hline 24 & M & 9 & 5 & Cong & $\mathrm{x}$ & & 5 & 9 & 0 & 3 & 0 & $\mathrm{~N}$ & 5 & 4 & 0 & $x$ & $x$ & 0 & 0 & 1 \\
\hline
\end{tabular}
and 2 were married. Two patients had become parents. (See Table 2 and 3 for full information of all patient data) 
Citation: Niemela BJ, Tjernstrom B (2014) Mended But Not Healed - Somatic and Mental Health 10 Years after Leg Lengthening with Ilizarov. J

Page 4 of 7

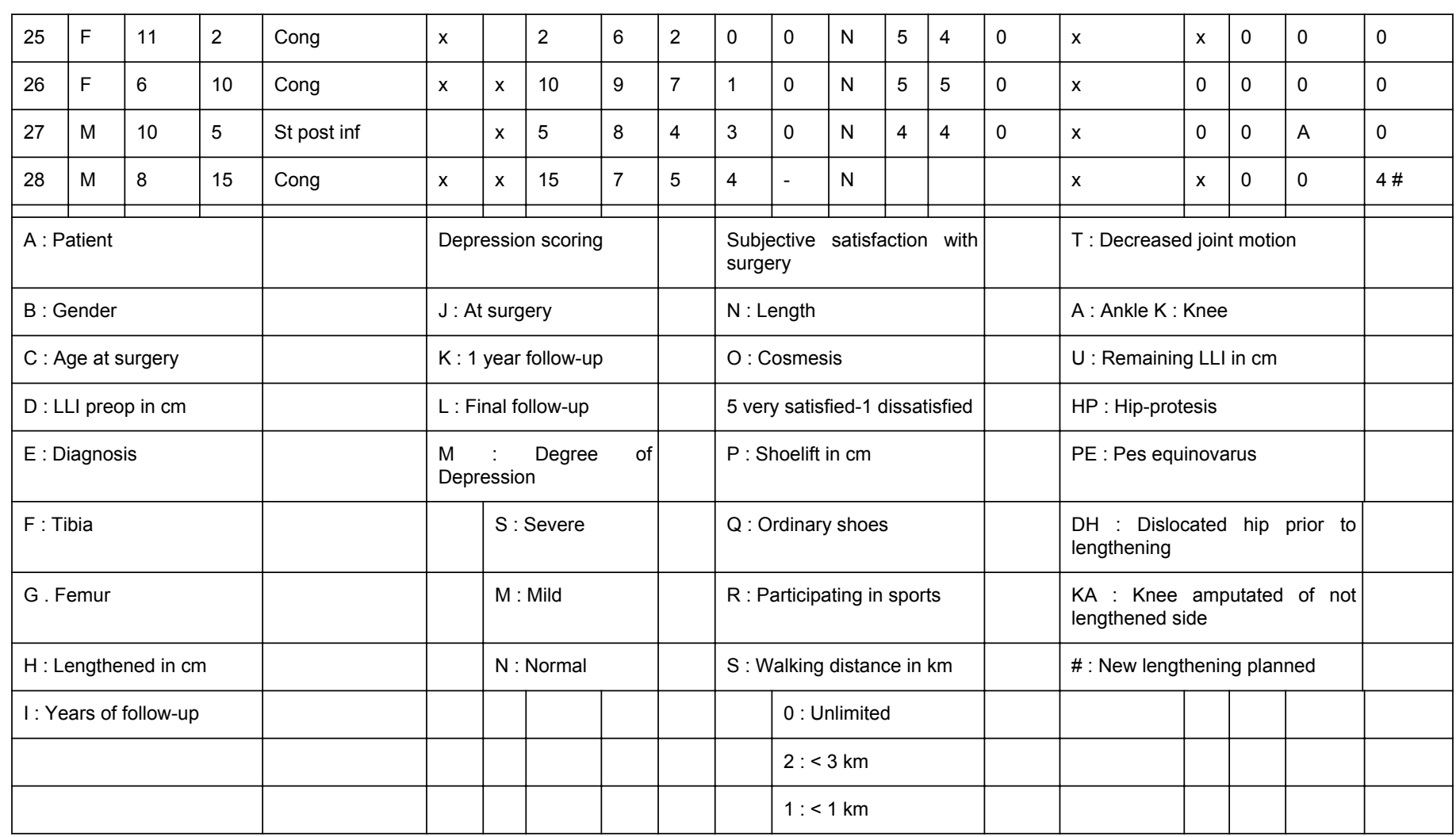

Table 3: All patient data

\section{Depressive symptoms}

Out of 7 patients in the 10-year follow-up, 5 were not depressed before or 1-year after surgery. One patient was mildly depressed before LL but had been recurred during the process of measurements. For the remaining depressed patient there was no pre-or 1-year follow-up.

At the 1-year follow-up 79\% (15/19) had improved their scores, 3 were worse and one had the same score. $Z=2.39$; $(p<0.05)[10]$. At the

\begin{tabular}{|l|l|l|l|}
\hline & $\begin{array}{l}\text { Pre op (CDI/ } \mathbf{n = 1 9 )} \\
(\mathbf{1 1} \text { had not answered) }\end{array}$ & $\mathbf{1 - y e a r ~ p o s t ~ o p ~ ( C D I / ~} \mathbf{n = 2 8 )}$ & $\mathbf{1 0 - y e a r ~ p o s t o p ~ ( B D I / ~} \mathbf{n = 2 7 )}$ \\
\hline Minimal & $17(89 \%)$ & $26(93 \%)$ & $20(74 \%)$ \\
\hline Mild & $1(5 \%)$ & & $4(15 \%)$ \\
\hline Moderate & $1(5 \%)$ & & \\
\hline Severe & & & $3(11 \%)$ \\
\hline
\end{tabular}

Table 4: Depressive symptoms

\section{Self-esteem}

When the patients retrospectively (at the 10- year follow-up) rated their self-esteem on a Likert scale (1-9) during the ages of 6 years $\mathrm{M}=$ 6.9 (SD 2.3), 8-10 years $\mathrm{M}=6.3$ (SD 2.3) and 13-19 years of age $\mathrm{M}=6.6$ (SD) 2.1 it was on a median level. In the previous study the patients' 10-year follow-up there were 17 patients to compare with the pre-op assessment. $13(76 \%)$ patients screened had no depression at either occasion, 4 (24\%) were screened depressed at 10-year follow-up, and one of those 4 had depression before surgery. $\mathrm{Z}=1.30(\mathrm{P}=0.19)$ (Table $4)$.

\begin{tabular}{|l|l|l|l|l|l|}
\hline & Baseline & $\mathbf{1}$ year follow-up & $\mathbf{1 0}$ years follow-up & & \\
\hline & Mean(sd) & Mean(sd) & Mean(sd) & $F(1,20)$ & $p$ \\
\hline
\end{tabular}


Citation: Niemela BJ, Tjernstrom B (2014) Mended But Not Healed - Somatic and Mental Health 10 Years after Leg Lengthening with Ilizarov. J Depress Anxiety 3: 151. doi:10.4172/2167-1044.1000151

Page 5 of 7

\begin{tabular}{|l|l|l|l|l|l|}
\hline State Anxiety $(\mathrm{n}=21)$ & $40.4(10.1)$ & $28.8(6.7)$ & $29.5(5.8)$ & 18.27 & $<.001$ \\
\hline Trait Anxiety $(\mathrm{n}=21)$ & $30.5(5.4)$ & $30.3(4.1)$ & $31.1(9.1)$ & 0.12 & 0.89 \\
\hline & age 6 & age 8-10 & age 13-19 & $\mathrm{F}(1,25)$ & \\
\hline Self-esteem $(\mathrm{n}=26)$ & $6.9(2.3)$ & $6.3(2.3)$ & $6.6(2.1)$ & 0.57 & 0.55 \\
\hline
\end{tabular}

Table 5: Development of Parents' State-Anxiety, Trait-Anxiety and Patients' Self-Esteem over time.Effect over time were tested with Repeated measure ANOVA with baseline as referens category.

\section{State- and Trait-Anxiety level}

Experience of anxiety and stress was measured by the STAI. Parents ' stress level, measured by the State Anxiety scale, in the immediate situation "being with their child/adolescent in the hospital" seemed to be normalized 1 year after $(\mathrm{F}=20.98 ; \mathrm{p}<0.001)$ as well as 10 years after LL $(\mathrm{F}=19.58 ; \mathrm{p}<0.001)$ compared to the extremely high stress experienced before LL. Trait-Anxiety level 1 year after $(\mathrm{F}=0.03 ; \mathrm{p}=0.86)$ and 10 years after LL $(\mathrm{F}=0.08 ; \mathrm{p}=0.77)$ was the same as before $\mathrm{LL}$ which is expected since it measures more of personality traits than the State-Anxiety. Parents' mean level State-Anxiety score was 40.4 (SD 10.1)-before LL, 28.8 (SD 6.7) 1 year after and 29.5 (SD 5.8) 10 years after. Parents' mean level Trait-Anxiety score 30.5 (SD 5.4), 30.3 (SD 4.1) and 31.1 (SD 9.1) (Table 5).

Patients' State- and Trait-Anxiety level is usually also related to depression and self-esteem and so was the case in the present study. Those who had reported depression scores demonstrated strong correlation to State $(r=0.91)$ and Trait $(r=0.83)$ stress on the State-Trait Anxiety Inventory (Figures $1 \mathrm{~A}$ and $1 \mathrm{~B}$ ).

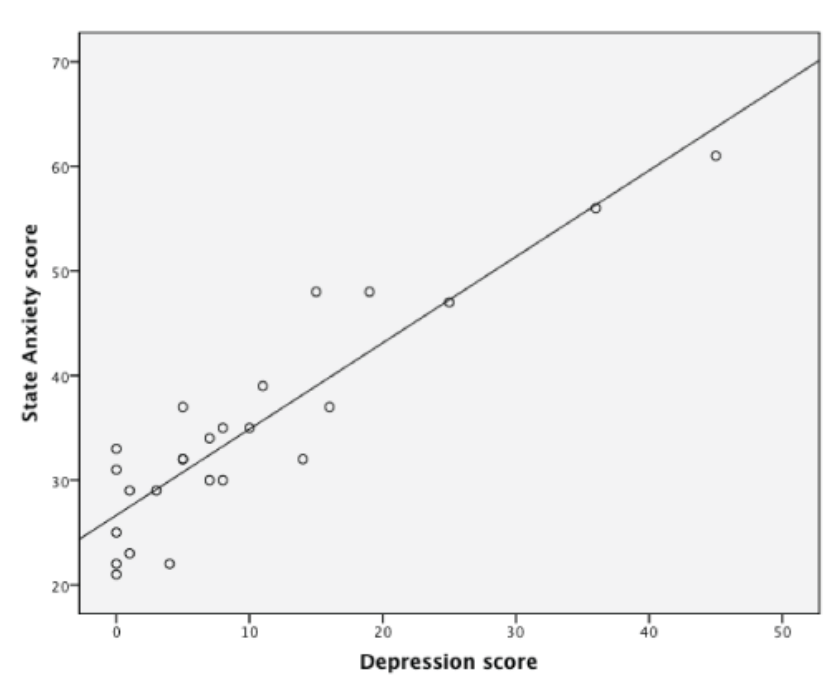

Figure 1A: Patients' State Anxiety and Depression Scores

There was no significant correlation between parents' and patients' State $(r=0.27 ; \mathrm{p}=0.21)$ or Trait score $(\mathrm{r}=0.33 ; \mathrm{p}=0.12)$ on a group level. There were no significant differences in patient age between girls $($ mean $=20.1 ; \mathrm{sd}=3.5)$ and boys $($ mean=20.6; $\mathrm{sd}=4.7)$ at time of surgery $(\mathrm{t}=0.27 ; \mathrm{p}=0.78)$.

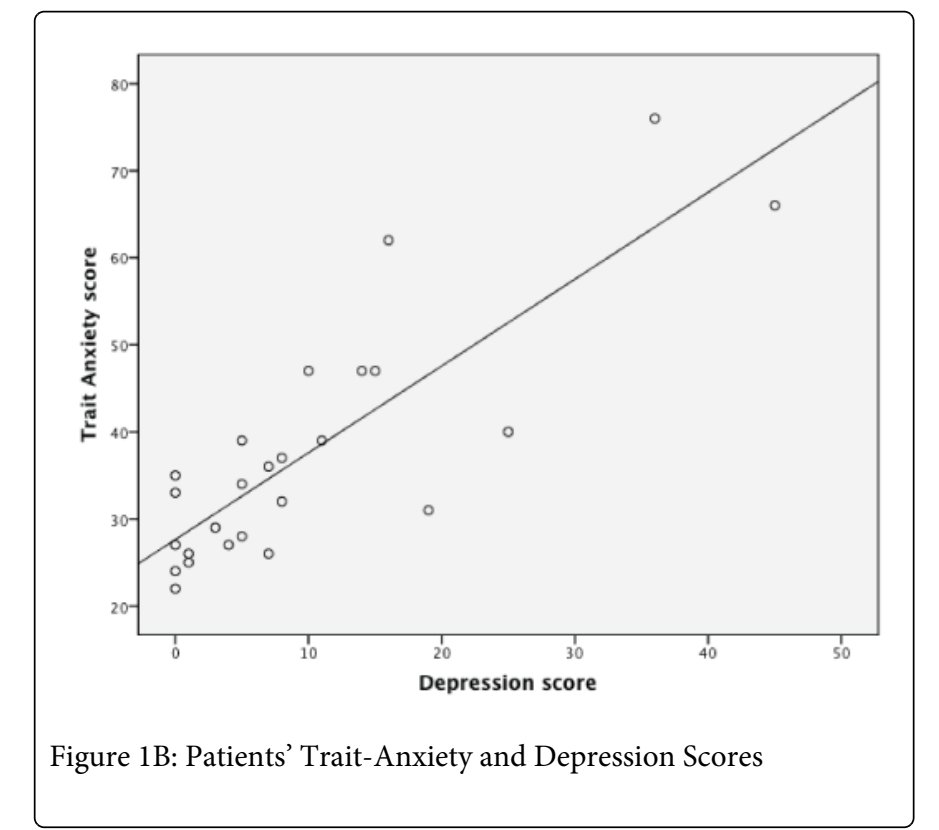

\section{Qualitative data- Condition specific topics - Interview data}

From the questionnaire Youth Questionnaire-Leg Length Inequality we learned that at an early age all patients had noticed that they were different from other children at preschool-elementary school age (3 -13 years). They experienced that they got a lot of attention because of LLI ( 7 patients answered none or a little. 21 answered a lot). On the question:

"How often do you think of your LLI?" 11 patients answered never or seldom, while 17 answered a lot or always. As for what emotional reactions patients remembered after surgery, the most frequent emotion reported was interest and hope for a better future by 20 patients. Sixteen patients reported being distressed. 6 had been angry and a few reported shame.

9 patients had a lot of pain, usually reported as pain when cleaning the pins. When patients reflected on their experiences of pain they said that it sometimes was difficult to differentiate from fear.

Patients' assessment of what made their self-esteem/view of themselves waver were their legs, the scars, the surgery and treatments, being in the school and the children there, meeting new peers, schooltrips, new classes and people wondering about LLI. 8 patients reported worse, 17 better and 3 the same level of self-esteem at the 10year follow-up. However only 5 patients thought they had been socially isolated because of LLI. 


\section{Discussion}

The psycho-social functioning of young patients undergoing LL has been frequently studied but studies on mental health and somatic function on a long term basis are sparse [11]. Our study was both prospective with a pre op assessment, a 1-year follow-up and a longterm study with a 10 -year follow-up of a cohort of LLI patients. We used standardized measures of somatic functioning and mental health. The parents were included in the study so as to gain more information on their state of anxiety to better understand their situation and how it could affect their childrens' mental health during the LL procedure. In order to detect more condition-specific problems the patients also filled in a specially designed questionnaire where they could look back and assess their self-esteem and social experiences during the process of LL [18].

Undergoing LL was a demanding procedure for this group of patients, in line with previous studies [6,7]. However the majority of patients were satisfied with the result of the LL as seen in the 10-year follow-up as well as the 1-year follow up [10]. Self-esteem continued to be on a median good level before, 1 year after and 10 years after surgery. It was quite suprising since self-esteem could be expected to decline during adolescence [12]. One fourth of the patients had symptoms of depression at the 10-year follow-up. Those who reported more depressive symptoms also had higher levels of anxiety, both the State-like and Trait-like. In order to better understand the dynamic factors affecting mental health, we tested for a correlation between patients' and parents' anxiety levels. However, there was no significant correlation between parents' and patients' State-or Trait score on a group level. There were no significant differences between gender or patient age at time of surgery. The mental health data at the 1-year and 10-year follow-ups demonstrate no clear connection.

In the individual cases we can see a connection between the severity of health problems such as the original disease, other major trauma sustained, remaining LLI and high levels of anxiety correlated to depression scores. The two patients who were amputated did not show signs of depression. On a group level there were no significant correlations between severity of health problems and depression. Most patients reported how the LL affected their lives; lack of understanding from schoolmates, difficulties taking part in sports, pain or negative emotions. The fact of not wanting to disturb or hurt their parents by talking of their own worries regarding people's attitudes, their own self-esteem and thoughts about the future could be depressing. The patients were satisfied with the medical care, but expressed a wish for sharing experiences in a group or talking individually with psychologists. We need more research on how reconstructive surgery affects self-esteem and mental health both from a developmental perspective and a body-mind perspective. Knowing about self-esteem development also has implications for the timing of LL.

The limitations with the study are that there is no control group of untreated LLI patients or patients with another orthopedic disorder, the difficulty of measuring and following experiences of depressive symtoms and anxiety in patient with different ages, as well as the cognitive capacity of adolescents to retrospectively answer questions about their self-esteem at different ages.

\section{Conclusion}

Reconstructive surgery by the Ilizarov technique does not pose a threat to the children's mental health according to a 1-year follow-up study. A follow-up 10 years after surgery demonstrates that the lengthening period was a period filled with strong feelings. Sorrow and fear were mixed with experiences of pain. Self-esteem was on a median good level. One fourth of the patients had depressive symptoms. Those with high level of depression scores also scored high on State- and Trait-Anxiety. However the group of LL patients was closer to a normal group than to a psychiatric sample. State and Trait anxiety levels were normal for the group of parents. There were no differences between gender or age of patient at the time of the surgery. Patients were satisfied with the medical care, but expressed a wish for sharing experiences in a group or having individual talks with psychologists. "It should be part of the treatment". From available data we could not conclude that the leg lengthening per se caused the depression in the seven patients.

\section{Acknowledgements}

B JN designed the study, performed the psychological assessments of the patients, analyzed the data, and wrote the manuscript. B T was involved in designing the study, assessing the patients and analyzing the orthopaedic data, contributing to the different drafts of the manuscript, as well as being involved in finalizing the manuscript. A special thanks to Hans Arinell for assistance with statistical analysis. We gratefully thank both adolescents and their parents for their participation in this study

\section{Funding:}

Uppsala University Hospital Fund did cover patients' travel costs.

\section{References}

1. Ilizarov GA (1989) The tension-stress effect on the genesis and growth of tissues. Part I. The influence of stability of fixation and soft-tissue preservation. Clin Orthop Relat Res : 249-281.

2. Ilizarov GA (1989) The tension-stress effect on the genesis and growth of tissues: Part II. The influence of the rate and frequency of distraction. Clin Orthop Relat Res : 263-285.

3. Herzenberg JE, Paley D (1998) Leg lengthening in children. Curr Opin Pediatr 10: 95-97.

4. Tjernström B, Olerud S, Rehnberg L (1994) Limb lengthening by callus distraction. Complications in 53 cases operated 1980-1991. Acta Orthop Scand 65: 447-455.

5. Birch JG, Samchukov ML (2004) Use of the Ilizarov method to correct lower limb deformities in children and adolescents. J Am Acad Orthop Surg 12: 144-154.

6. Hrutcay JM, Eilert RE (1990) Operative lengthening of the lower extremity and associated psychological aspects: The children's hospital experience. J Pedriatric Orthop 3: 373-377.

7. Maffuli N, Fixsen JA (1996) Distraction osteogenesis in congenital limb length discrepancy: a review. J R Coll Surg Edinb 41: 258-264.

8. Ramaker RR, Lagro SW, van Roermund PM, Sinnema G (2000) The psychological and social functioning of 14 children and 12 adolescents after Ilizarov leg lengthening. Acta Orthop Scand 71: 55-59.

9. Martin L, Farrell M, Lambrenos K, Nayagam D (2003) Living with the Ilizarov frame: adolescent perceptions. J Adv Nurs 43: 478-487.

10. Niemelä BJ, Tjernström B, Andersson G, Wahlsten VS (2007) Does leg lengthening pose a threat to a child's mental health?: An interim report one year after surgery. J Pediatr Orthop 27: 611-617.

11. Moraal JM, Elzinga-Plomp A, Jongmans MJ, Roermund PM, Flikweert PE, et al. (2009) Long-term psychosocial functioning after Ilizarov limb lengthening during childhood. Acta Orthop 80: 704-710.

12. Robins RW, Trzniewski KH (2005) Self-Esteem Development Across the Lifespan. Current Directions in Psychological Science 14: 158-162. 
Citation: Niemela BJ, Tjernstrom B (2014) Mended But Not Healed - Somatic and Mental Health 10 Years after Leg Lengthening with Ilizarov. J Depress Anxiety 3: 151. doi:10.4172/2167-1044.1000151

Page 7 of 7

13. Auerbach SM (1973) Trait-state anxiety and adjustment to surgery. J Consult Clin Psychol 40: 264-271.

14. Spielberger CD, Auerbach SM, Wadsworth AP, Dunn TM, Taulbee ES (1973) Emotional reactions to surgery. J Consult Clin Psychol 40: 33-38.

15. Spielberg CD (1983) State-Trait Anxiety Inventory. Mind garden, Redwood City, California.

16. Searcy YD (2007) Placing the horse in front of the Wagon: Toward a conceptual understanding of the development of self-esteem in children and adolescents. Child and adolescent Social work journal 24: 121-131.
17. Beck AT, Steer RA, Brown GK (2008) Manual for the Beck Depression Inventory- Second Edition. Harcourt Assessment.

18. Niemelä JB (2008) Mental Health in Children Undergoing Reconstructive Surgery. Doctorial Thesis, Uppsala University. 